\title{
Biological Nanopore Probe: Probing of Viscous Solutions in a Confined Nanospace
}

\author{
Masaki Matsushital, Kan Shoji ${ }^{1,2,3}$, Natsumi Takai ${ }^{1}$, and Ryuji Kawano ${ }^{1, *}$
}

${ }^{1}$ Department of Biotechnology and Life Science, Tokyo University of Agriculture and Technology, Japan

${ }^{2}$ Department of Chemistry, University of Cincinnati, USA.

${ }^{3}$ Department of Mechanical Engineering, Nagaoka University of Technology, Japan

\section{Fabrication of nanoneedle}

A carbon rod with $3 \mathrm{~mm}$ diameter was used as a counter electrode, and a function generator (GFG-8219A; GW Insteck) was used to control the input voltage magnitude and frequency. The $1 \mathrm{~mm}$ tip of the gold wire was vertically immersed in the $\mathrm{CaCl}_{2}$ solution. Then, a sine wave AC voltage was applied between the gold wire and the counter electrode until the current reached zero typically in $60 \mathrm{~min}$. The typical conditions of the applied voltage were AC voltage of 12 Vpeak to peak (p-p) at $10 \mathrm{kHz}$ with a DC offset of $9.5 \mathrm{~V}$.

Fabrication of nanopore probe using gold nanoneedle. Gold nanoneedle-based nanopore probes were prepared as described previously. ${ }^{1-2}$ Gold nanoneedles were fabricated by electrochemical etching of gold wires with $0.25 \mathrm{~mm}$ diameter in a $2.2 \mathrm{M}$ 
$\mathrm{CaCl}_{2}$ solution. (See supporting information for details of the conditions of electrochemical etching.) The nanoneedle was modified with thiol-PEG to prepare a hydrophilic surface. First, the gold nanoneedle was electrochemically cleaned to prepare a fine gold surface. Then, the tip of the gold nanoneedle was immersed in $60 \mathrm{mg} / \mathrm{mL}$ thiolPEG solution (in ethanol) for $2 \mathrm{~h}$ at room temperature. Finally, the nanoneedle was rinsed with ethanol and deionized water.

A layered bath solution of the aqueous electrolyte solution $\left(0.2 \mathrm{M} \mathrm{KNO}_{3}, 20 \mathrm{mM}\right.$ sodium phosphate buffer, $\mathrm{pH}$ 7.4) with $10 \mathrm{nM} \alpha \mathrm{HL}$ and $1 \mu \mathrm{M}$ hpDNA and the lipid/oil mixture (20 mg/mL DPhPC in n-decane) was prepared. The PEG-modified gold nanoneedle was dipped in the $1 \mathrm{M} \mathrm{KNO}_{3}$ solution (20 $\mathrm{mM}$ sodium phosphate buffer, $\mathrm{pH}$ 7.4) for $10 \mathrm{~min}$ and then immersed in the bath solution. A BLM is formed on the tip by contacting lipid monolayers formed on the surface of the nanoneedle and the interface of the aqueous solution and the lipid/oil mixture when the tip is pushed through the interface of these two layers.

Construction of the nanopore probe using a gold nanoneedle. We attempt to develop a practical probing system using a gold nanoneedle. We have recently developed the use of an etched gold wire modified with a PEG monolayer as a support for a lipid bilayer and subsequent nanopore measurements with spatial resolution (Figure S2). ${ }^{3}$ The hpDNA can also be inserted into the $\alpha \mathrm{HL}$ pore in the gold nanoneedle system. A single blocking state that means the hpDNA does not move in the pore was observed in this system probably due to the different salt concentration profile (see Experimental section). In the nanoneedle system, lower salt concentrations were used to prevent the undesired current decay caused by charging up of the gold nanoneedle. The lower salt concentration 
provided the condition for the salting in effect, and consequently the hydrogen bond strength was enhanced. Therefore, the single current blocking state which may correspond to the $\mathbf{L L}$ state was observed. To combine the nanoneedle-based nanopore probe for the spatially resolved analysis of solution conditions, we used an equilibrium electrode, such as $\mathrm{Ag} / \mathrm{AgCl}$, for preventing current decay without changing the salt concentration profile. 

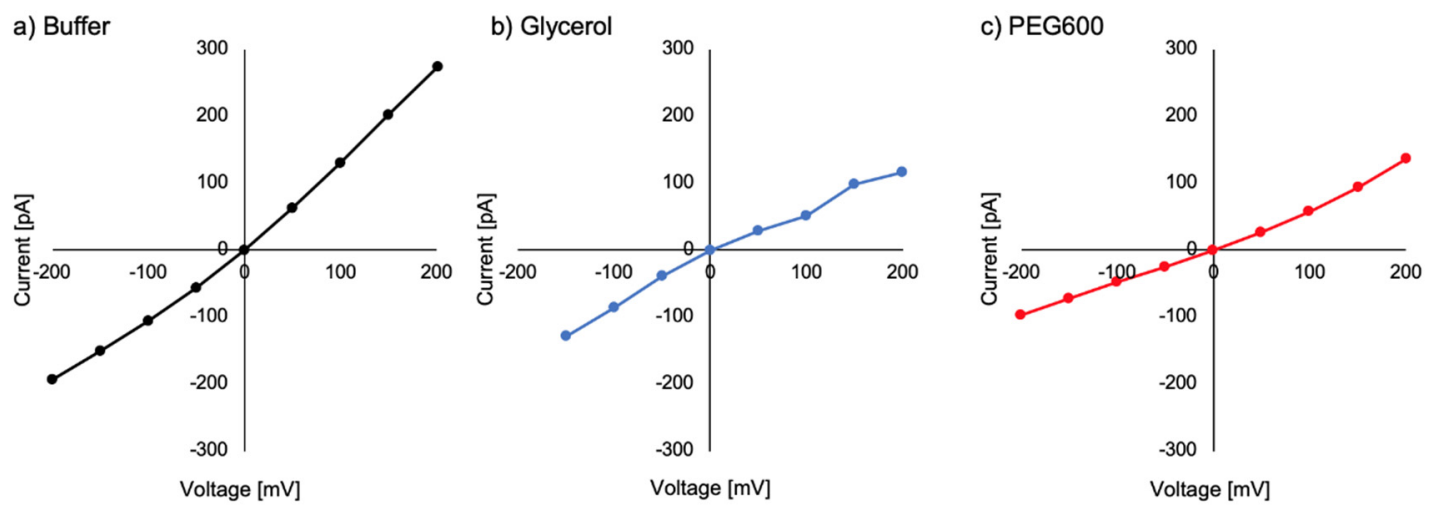

Figure S1 Current and voltage curves of (a) buffer (1.0 mPa s), (b) glycerol (2.0 mPa s), and (c) PEG600 (2.0 mPa s) solution. 
a)
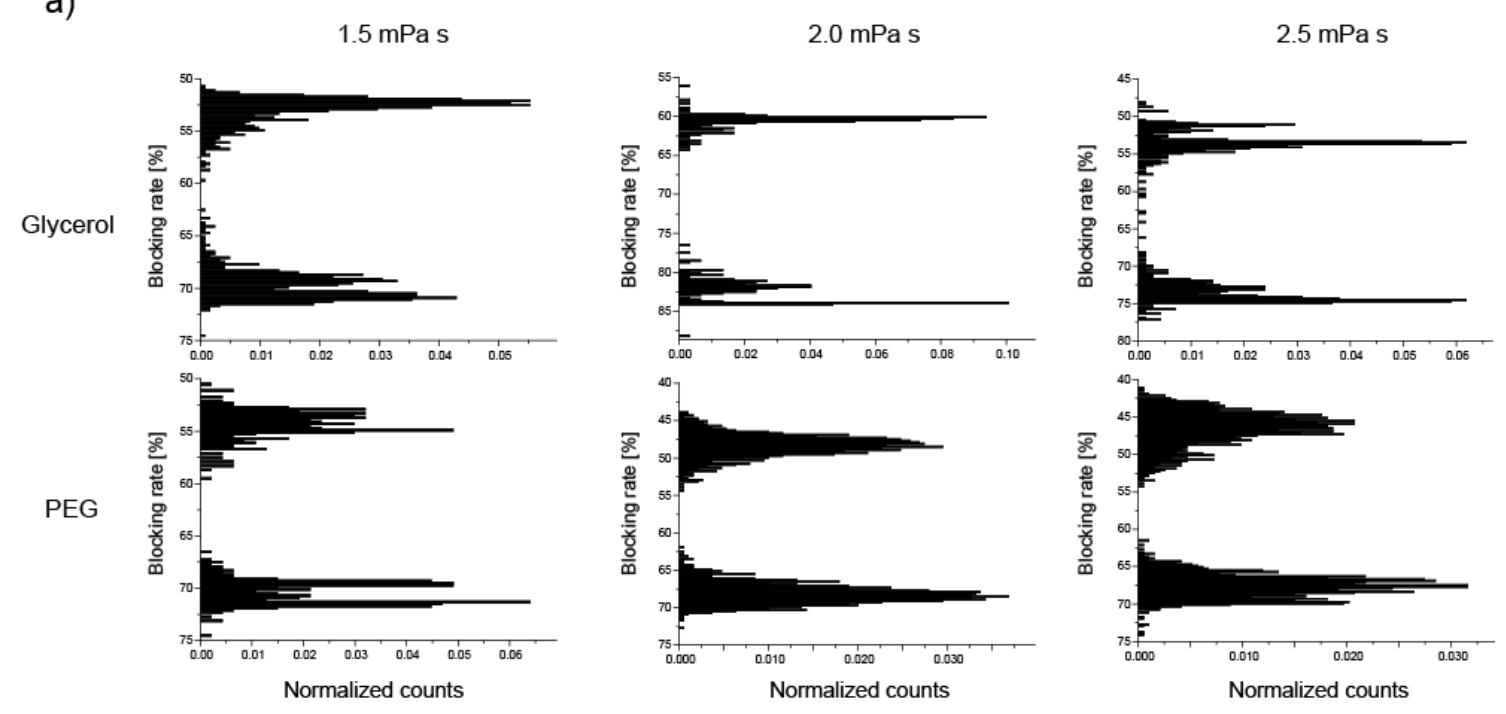

b)
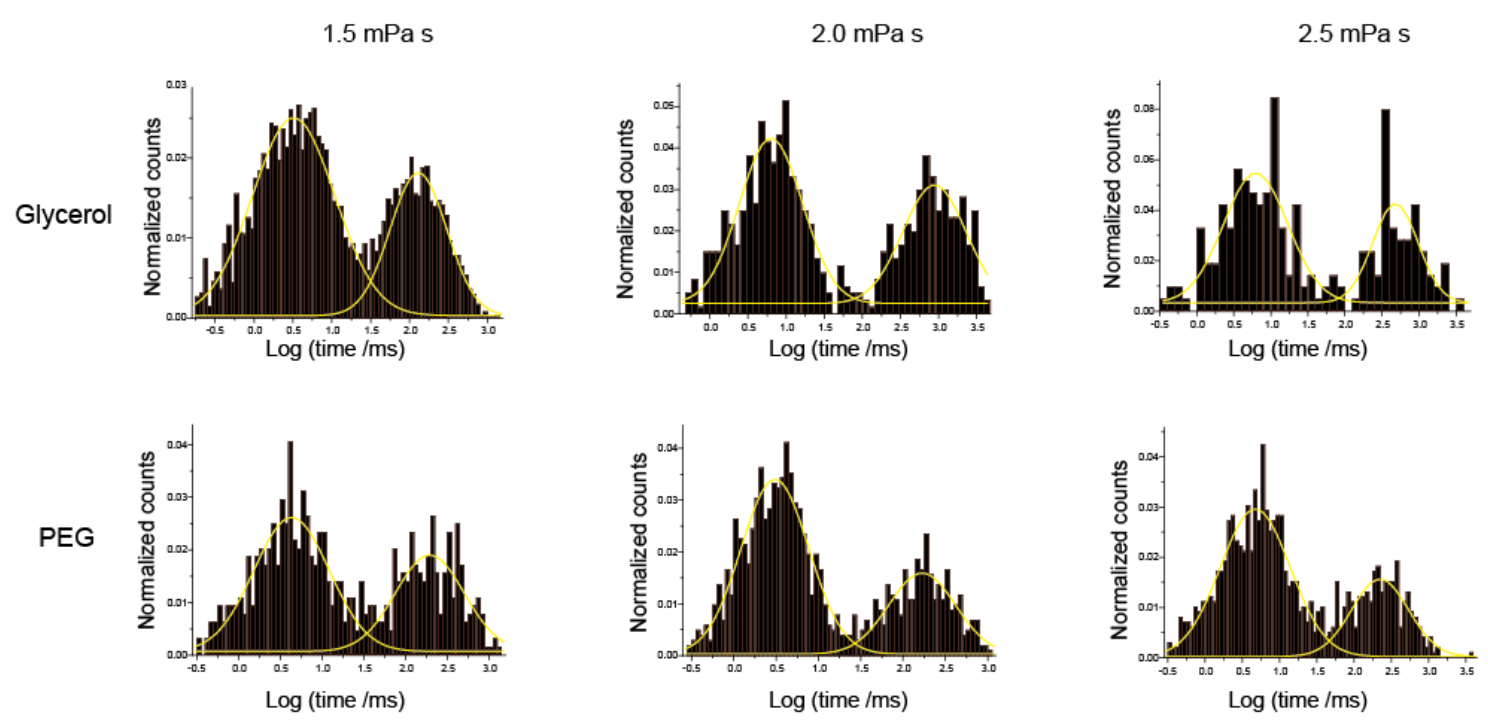

Figure S2 Histograms of the current blockings and the duration time. (a) The histograms of the current blockings of hpDNA in each condition. The UL, IL, and LL states were determined by picking the peak top using Origin software. (b) The histograms of duration time of IL and LL. When these two states were unseparated in the blocking current analysis, we analyzed to determine each state using the duration time. 
a)

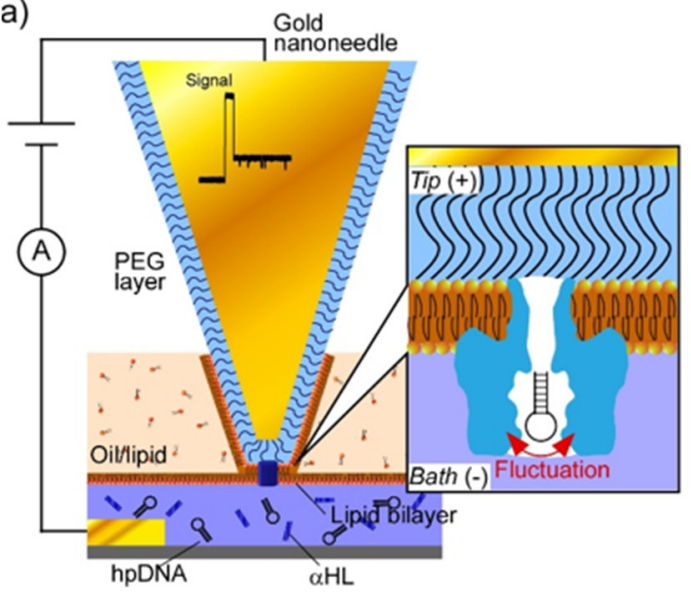

b)

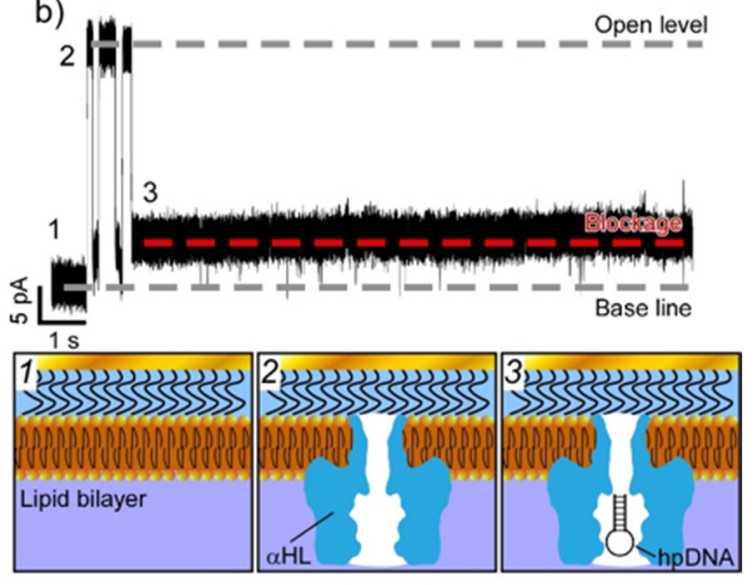

Figure S3 (a) Schematic illustration of a nanopore probe using a gold nanoneedle. An $\alpha H L$ nanopore is reconstituted into a BLM formed on a tip of the gold nanoneedle, and a hpDNA is inserted into the $\alpha \mathrm{HL}$ pore. (b) Recorded channel current and schemes of nanopore formation and hpDNA insertion expected from the current signals. 


\section{References}

1. Okuno, D.; Hirano, M.; Yokota, H.; Onishi, Y.; Ichinose, J.; Ide, T., A Simple Method for Ion Channel Recordings Using Fine Gold Electrode. Anal. Sci. 2016, 32 (12), 1353-1357.

2. Okuno, D.; Hirano, M.; Yokota, H.; Ichinose, J.; Kira, T.; Hijiya, T.; Uozumi, C.; Yamakami, M.; Ide, T., A gold nano-electrode for single ion channel recordings. Nanoscale 2018, 10 (8), 4036-4040.

3. Shoji, K.; Kawano, R.; White, R. J., Spatially Resolved Chemical Detection with a NanoneedleProbe-Supported Biological Nanopore. ACS Nano 2019, 13 (2), 2606-2614. 